\title{
Cooperative Power Saving Strategies for IP-Services Supported over DVB-H Networks
}

\author{
Zhang, Qi; Fitzek, F.H.P.; Katz, Marcos
}

Published in:

IEEE Wireless Communications and Networking Conference, 2007. WCNC 2007.

Link to article, DOI:

10.1109/WCNC.2007.750

Publication date:

2007

Document Version

Publisher's PDF, also known as Version of record

Link back to DTU Orbit

Citation (APA):

Zhang, Q., Fitzek, F. H. P., \& Katz, M. (2007). Cooperative Power Saving Strategies for IP-Services Supported over DVB-H Networks. In IEEE Wireless Communications and Networking Conference, 2007. WCNC 2007. IEEE. https://doi.org/10.1109/WCNC.2007.750

\section{General rights}

Copyright and moral rights for the publications made accessible in the public portal are retained by the authors and/or other copyright owners and it is a condition of accessing publications that users recognise and abide by the legal requirements associated with these rights.

- Users may download and print one copy of any publication from the public portal for the purpose of private study or research.

- You may not further distribute the material or use it for any profit-making activity or commercial gain

- You may freely distribute the URL identifying the publication in the public portal 


\section{Cooperative Power Saving Strategies for IP-Services Supported over DVB-H Networks}

\author{
Qi Zhang \\ Department of Communication, \\ Optics \& Materials \\ Technical University of Denmark \\ Building 343, DK-2800 \\ Kgs. Lyngby, Denmark \\ Email:qz@com.dtu.dk
}

\author{
Frank H.P. Fitzek \\ Department of Communications \\ Technology \\ Aalborg University \\ Neils Jernes Vej 12, 9220 \\ Aalborg Øst, Denmark \\ Email: ff@kom.aau.dk
}

\author{
Marcos Katz \\ VTT Technical Research \\ Centre of Finland \\ P.O.Box 1100 \\ FI-90571 Oulu \\ Finland \\ Email:marcos.katz@vtt.fi
}

\begin{abstract}
This paper introduces power saving strategies for cooperative wireless communication systems. The described scenario focuses on IP-services over DVB-H networks showing the strength of non-altruistic cooperation between mobile devices. The envisioned cooperation is based on cellular reception of data, which is then shared among mobile devices within each others' proximity over short-range links. As the state-of-the-art, we use Bluetooth technology for the short-range link communication in this cooperative scheme. In this paper, three topology based cooperative algorithms for the short-range link communication are designed. Then numerical results show that a power saving gain of over $50 \%$ can be achieved by cooperative networking of three mobile terminals in fully cooperating mode.
\end{abstract}

\section{INTRODUCTION}

The aim of the Digital Video Broadcasting on Handheld (DVB-H) standard is to deliver audio and video content to mobile handheld devices. As those devices are battery driven, power is always a crucial issue for mobile application. So far time slicing [2][4] has been introduced into DVB-H to save power. The basic idea behind time slicing is to convey data in bursts with long pause periods in between instead of sending a steady low data rate stream. The power consumption with time slicing depends on the burst duration and the so-called OFF-time period. From a power saving perspective, the shorter the burst duration is, the more power can be saved. However, because of receiver sensitivity performance constraint, the burst duration is increased to reduce the sensitivity of the receiver. Thus, the remaining factor to work on is the OFFtime period. Obviously, the longer OFF-time period results in the more power saving. Unfortunately the OFF-time period can not be excessively long because of quality of service aspects such as the access time and zapping time ${ }^{1}$. Therefore, there is clearly a trade-off between burst duration and OFF-time to have optimum service access time and power consumption.

IP-services over DVB-H can be transmitted in sequential elementary streams (SESs) or parallel elementary streams (PESs). Both types of streams are transmitted in a multicast or broadcast fashion. The SESs carry one service in one burst, while PESs carry multiple services in one burst. The

\footnotetext{
${ }^{1}$ Zapping time means the program or channel switching time.
}

reason that multiple services are bundled in the same stream and transported within the same burst is that the burst needs to meet a minimum length to reduce the sensitivity of the receiver and at the same time the DVB-H system tries to get the maximal utilization of the DVB-H bandwidth. The use of parallel elementary streams brings many benefits, for instance, zapping time reduction, bandwidth optimization, the possibility of sending message type services in parallel to the main services, etc. However, when implementing PESs, the energy leak becomes an issue because the mobile terminal receives parallel elementary streams of all the services in the same burst. Indeed, it only keeps the desired elementary streams discarding the remaining ones. From the entire system or network standpoint, the elementary streams discarded by a given terminal could be used by other mobile terminals. It is clear that discarding unwanted elementary streams leads to inefficient use of resources, particularly power. To our knowledge, this issue has not been addressed before. Furthermore as pointed out in [5], more research is needed in terms of novel techniques aiming at reducing power consumption. In this direction, time slicing techniques have been recently introduced. End users generally expect more and more hours of streaming audio on one battery charge but, on the other hand, improvements in battery capacity develop slowly (typically $10 \%$ per year) and hence, the requirements are difficult to meet. Power saving techniques like those considered here are a promising option to extend considerably the service time of terminals.

In this paper we propose a cooperative power saving strategy for IP-services over DVB-H. The considered cooperative strategy focuses on reducing energy consumption in the PESs case, even though it can be as well applied to SESs to obtain further power saving gains. The cooperative architecture is set up between mobile devices that are capable to communicate not only with a central base station but also among each other using short-range wireless technology. The essential reason of this approach achieving power saving gain is that by cooperative reception of data over cellular link, the OFF time period is virtually increased. Furthermore, as the energy per bit is much lower on a short-range link than in a cellular 
one, the power consumption overhead over the former is very low.

In [6] the power saving issue in a similar scenario is addressed. However, the approach there saves energy by leaving out some FEC columns in the MPE-FEC (MultiProtocol Encapsulation-Forward Error Correction) frame once the receivers have received all the error free data packets instead of getting the full block always. Its maximum power saving gain is $25 \%$. Our proposed cooperative power saving strategy not only has much larger power saving potential than that of [6] but also can be built on top of that.

This paper is organized as follows. Section II describes the cooperative scheme for parallel elementary stream services. Section III proposes the design of the short-range link cooperative mechanisms and the topology based cooperative algorithms. Section IV gives two numerical examples and corresponding analysis. A conclusion is reached in Section V.

\section{CoOperative Strategy For IP-Services OVER DVB-H}

Cooperation is the strategy of a group of entities working together to achieve a common and/or individual goal [1]. The proposed cooperative mechanism requires that the terminals have two air interfaces: a cellular link (CL) for DVB-H packets reception and a short-range link (SRL) for exchanging packets locally. The basic idea of the cooperative mechanism is that terminals cooperatively receive the DVB-H bursts. Each cooperative node only receives partially the data over CL. In case of PESs, the mobile terminal does not discard the unwanted packets anymore, but forwards those packets to its cooperative peers. By reciprocity, it gets its missed packets from those peers. As for services by SESs case, peers simply exchange the missed packets using SRL. This scheme is beneficial to reduce the energy consumption since the energy per bit is much less in the short-range communication system. Furthermore, it guarantees very short service access time and zapping time. It does not require any modification in current DVB-H standard. The short-range link communication is easily implemented in the mobile terminals. The probability of having cooperative peers in one's proximity is quite high, specially in public places such as airports, bars, train stations, schoolchildren playgrounds. But the detailed analysis of marketing scenarios and potentials is out of the scope of this paper.

For multi-services transmitted by PESs scenario, the cooperative strategy works as following. We assume that there are three mobile terminals which are interested in three different services with the same data rate transmitted by PESs, respectively ${ }^{2}$. Base station transmits DVB-H bursts as usual without awareness of cooperation. But the mobile terminals can autonomously receive DVB-H bursts alternatively, if they are willing to cooperate after their negotiation. For instance, after MT1 finishes reception of the first burst containing the three services, it goes into sleep on its CL. And it transmits the

\footnotetext{
${ }^{2}$ Mobile terminals (MT1, MT2, MT3) are interested in service 1, 2, 3, respectively.
}

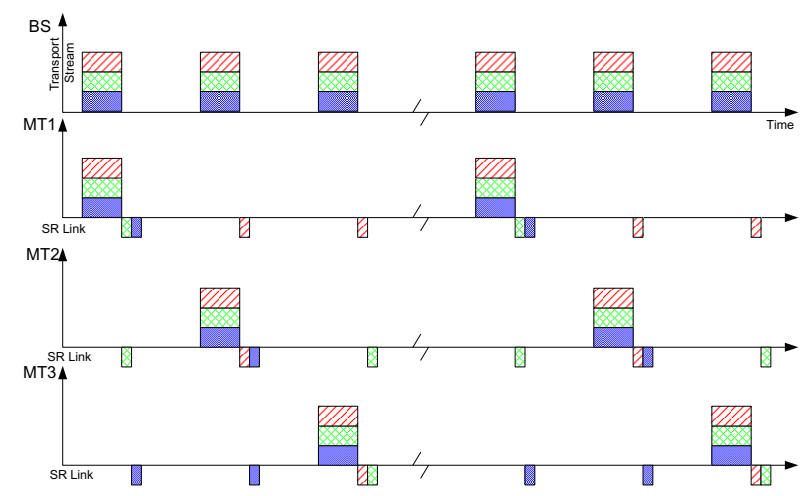

Fig. 1. Burst Flow of Cooperative Mechanism in Parallel Elementary Streams

related packets to MT2 and MT3 on SRL, respectively. Then MT2 and MT3 wake up at the start of the second and the third DVB-H burst respectively. They deal with the packets in a similar way as MT1 does. After a cyclic period, MT1 wakes up again to receive the forth burst, and so forth. Therefore, MT1 always wakes up at the start of the $(3 n+1)$ th burst. Fig. 1 illustrates how this cooperative mechanism works. The proposed cooperative strategy achieves power saving gain by longer idle time on DVB-H link. Furthermore, it also saves the power consumption that is spent on decoding the received MPE-FEC frame ${ }^{3}$.

In the above example, the three different services have the same data rate so each terminal receives one DVB-H burst every three bursts. In reality the different services might have different data rates. So the frequency of node waking up can be adjusted flexibly. This could be typically negotiated among the mobile terminals before cooperation starts. Thus the power consumption of all mobile terminals is balanced.

SESs can be regarded as the simplest case of PESs. It is obvious that the cooperative strategy can easily be adapted to the services transmitted by SESs.

Whether to cooperate or not should be evaluated by each mobile device independently. The decision depends on the cooperation strategy and the neighboring devices. In short, cooperation should be established as soon as the individual mobile device sees it own advantages [1]. It means that the establishment and termination of cooperation between mobile terminals depends on the goal of the involved terminals, the cooperative strategy in use, and the prevailing relationship among terminals. The relationships among terminals change with time as terminals move, terminate an ongoing service, join the network, their associated channels change, etc. For instance, in the above scenario, they will cooperate if they are close enough to each other to get mutual power saving gain. However, if a node cannot attain power saving gain anymore because of nodes movement, it will stop cooperation right away.

\footnotetext{
${ }^{3}$ The decoded packets are exchanged directly between peers without multiprotocol encapsulation and Reed-solomon coding
} 


\section{Designing Short-RAnge Link Communication Mechanisms SupPorting CoOPERATIVE StRategies}

According to the above description of the cooperative communication mechanism, the SRL is required to be very flexible and transparent to the end users. It works without any infrastructure in an autonomous mode. Namely, the shortrange connection is a sort of ad hoc connection based on peer communications. The SRL air interface and associated communication mechanism can be designed and implemented by many different approaches, only if it meets the cooperative strategy principles. We use Bluetooth technology (Bluetooth 2.0 EDR) as an example to illustrate how it supports cooperation strategy in the short-range link, though any other shortrange communication technology can be also used.

We define cooperation range as the range within which the nodes can achieve power saving gain. Every node has its own cooperation range. A node is capable of discovery services and has possibility to cooperate with the discovered peers within its cooperation range. When the node cooperates with peer nodes in its cooperation range, they form a cooperative piconet. In the cooperative piconet, which role (Master/Slave) to take or which cooperative approach (centralized or distributed approach) to use is dependent on the topology of the formed piconet or scatternet.

\section{A. Topology Based Cooperative Algorithm}

The cooperative algorithms in the short-range link can be summarized into the following three basic approaches according to the topologies.

1) Piconet Based Centralized Cooperative Approach: This approach is used for Topology I (see Fig. 2(a)). In this topology all wireless terminals form one piconet. The slaves within the piconet are out of each others' cooperation range. In this topology, the master controls the slaves' states and transmission slots as typical Bluetooth piconet. But the slaves all stay in PARK state in the most of time. When the master wishes to transmit the received DVB-H packets to its cooperative slaves, it will unpark slaves by a master-initiated unparking method (using dedicated link manager protocol unpark command with slaves PM_ADDR or its BD_ADDR). A slave can also unpark itself when it needs to transmit the received DVB-H packets to the master by a slave-initiated unparking method (sending access request message with AR_ADDR).

2) Piconet Based Distributed Cooperative Approach: This approach is used for Topology II (see Fig. 2(b)). In topology II the wireless terminals form one piconet and all the nodes are within each others' cooperation range. Namely, they can have fully meshed connections. In such case, all the nodes within the piconet are capable to work as a master and they alternatively take master role in the piconet. Therefore, all the nodes periodically switch role (Master/Slave) and only master node transmits in its turn. When the first master establishes the cooperative piconet, it decides the master role switching sequence and broadcasts it to its slaves. The key technical issue here is efficient role switching which can be initiated by either slave or master. The master initiated role switching

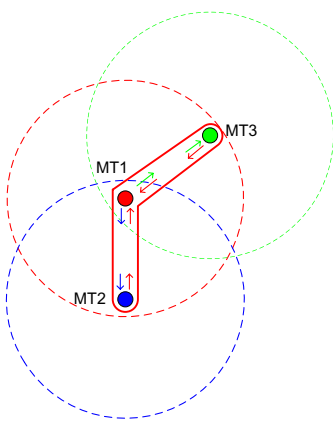

(a) Topology I

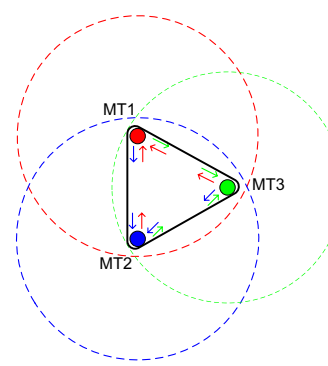

(b) Topology II

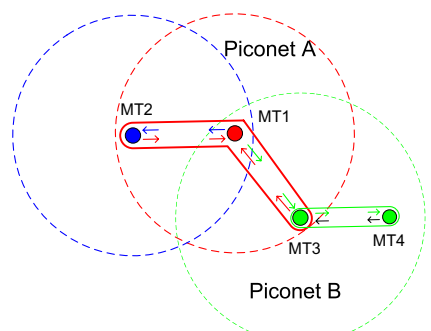

(c) Topology III

Fig. 2. Cooperative Topology

method is preferred, because it can implicitly check if the slave (i.e., the successor master) is still in the piconet or not. If the slave (i.e., the successor master) has gone, the master can timely update the master role switching sequence. Then it will switch role with another successor in the new role switching sequence list. The timely update can also effectively prevent free rider from cheating in the cooperative group.

3) Scatternet Based Cooperative Approach: This approach is employed for Topology III (see Fig. 2(c)). In topology III the wireless terminals form a scatternet, i.e., some nodes stay in more than one piconets. Considering signalling complexity and the achievable cooperation gain, we restrict the node can work at the most in two piconets currently. In Fig. 2(c) MT3 can work as slave in both Piconet A and B or it can work as slave in the Piconet A and master in the Piconet B. MT3 should be able to harmonize its operation in both piconets. For instance, MT3 works as master in the Piconet B during its PARK state interval in Piconet A. It requires MT3 to have an accurate synchronization in Piconet A, otherwise it might miss the unpark message from master of Piconet A. This issue can be effectively resolved by synchronization knowledge from DVB-H system.

\section{NumericAl EXAMPles FOR POWER CONSUMPTION ANALYSIS}

This section will give two numerical examples to illustrate power saving gain by cooperation on the short-range link.

The average power consumption without cooperation and with cooperation are expressed as Eq. 1 and Eq. 2, respectively. The notation of parameter expressions are given in Table I. 
TABLE I

$P_{\text {nocoop }}=\frac{\left(t_{c, B d}+t_{c, s y n}+\frac{t_{c, D j}}{2}\right) P_{c, o n}+t_{c, o f f} P_{c, o f f}+t_{c, i} P_{c, i}}{t_{c, c y c}}$

where, $\quad t_{c, c y c}=t_{c, B d}+t_{c, s y n}+t_{c, D j} / 2+t_{c, o f f}+t_{c, i}$

$$
\begin{aligned}
P_{\text {coop }}= & \left(t_{c, o n} P_{c, \text { on }}+t_{c, \text { of } f} P_{c, o f f}+t_{c, i} P_{c, i}\right) / t_{c, c y c}^{c o o p}+ \\
& \left(t_{s r, t x} P_{s r, t x}+t_{s r, r c} P_{s r, r c}+t_{s r, i} P_{s r, i}\right) / t_{s r, c y c}^{c o o p}
\end{aligned}
$$

where,

$$
\begin{aligned}
t_{c, o n} & =t_{c, B d}+t_{c, s y n}+t_{c, D j} / 2 \\
t_{c, c y c}^{c o o p} & =t_{c, o n}+t_{c, o f f}+t_{c, i} \\
t_{s r, c y c}^{c o o p} & =t_{s r, t x}+t_{s r, r c}+t_{s r, i} \\
t_{c, c y c}^{c o o p} & =t_{s r, c y c}^{c o o p}
\end{aligned}
$$

In Eq. $2 t_{c, i}$ is much longer than that in Eq. 1. Consequently $t_{c, c y c}^{c o o p}$ is much longer than $t_{c, c y c}$.

The first example is that nodes have full connections as Topology II in Section III. In this example let us assume that SRL has power control. Transmission power is a function of distance between transmitter and receiver. Due to the complexity of power control mechanism, the exact expression of transmission power is unknown. We just assume that transmission power is roughly proportional to the distance between peers. It is also assumed that the SRL has very short synchronization time. The transmission data rate on the SRL for master is $1.3 \mathrm{Mbps}$ (with 3-DH5 packet symmetrical maximum rate)[7]. ${ }^{4}$ We set the reception power consumption and idle power consumption to fixed values as $10 \mathrm{~mW}(10 \mathrm{dBm})$ and $1 \mathrm{~mW}$ $(0 \mathrm{dBm})$ [7], respectively. Transmission power consumption is varying between range of $10 \mathrm{~mW}-100 \mathrm{~mW}(10 \mathrm{dBm}-20 \mathrm{dBm})$ on SRL. The values of the parameters on the CL referred to [2] are listed in Table I. With all these assumptions, the relation of average power saving gain $\left(1-P_{\text {coop }} / P_{\text {nocoop }}\right)$ and transmission power is shown in Fig. 3. It can be seen from Fig. 3 that the achievable power saving gain is over $50 \%$ when three nodes fully cooperate.

The second example scenario is that three mobile terminals (MT) are receiving three different services (with same data rate), individually. The power consumptions of transmission, reception and idle state are assumed to be constant. The positions of two mobile terminals (MT1 and MT2) are fixed and they form a piconet. Another mobile terminal (MT3) is moving from far away to these two mobile terminals, then it moves away again. Fig. 4 illustrates the topology of the scenario and MT3's trace of this example. The parameters used in this example is listed in Table II.

The calculation results of this example is shown in Fig. 5. Two different cooperative strategies are implemented for nonfully meshed cooperative situation, i.e., Topology I scenario. Fig. 5(a) is generated by the Cooperation Strategy I which considers the selfish characteristics of nodes and the fairness

\footnotetext{
${ }^{4}$ 3-DH5 packet type is newly defined in Enhanced Date operation. 3-DH5 has maximum payload of 1021 Bytes, occupying 5 time slots.
}

PARAMETER LIST

\begin{tabular}{|c|c|c|}
\hline \multicolumn{3}{|c|}{ Cellular link Parameters } \\
\hline Character & Mean & Value \\
\hline$P_{c, o n}$ & power consumption when RF is on & $400 \mathrm{~mW}$ \\
$P_{c, \text { of } f}$ & RF is shut down, but MPE-FEC is on going & $50 \mathrm{~mW}$ \\
$P_{c, i}$ & DVB-H receiver waiting for next burst & $10 \mathrm{~mW}$ \\
$t_{c, s y n}$ & synchronization time & $120 \mathrm{~ms}$ \\
$t_{c, B d}$ & Burst duration & $236 \mathrm{~ms}$ \\
$t_{c, D j}$ & Delta-t jitter & $10 \mathrm{~ms}$ \\
$t_{c, o f f}$ & Time when receiver is at RF_OFF state & $500 \mathrm{~ms}$ \\
$t_{c, i}$ & Idle time & - \\
$t_{c, c y c}$ & Frame cyclic period without cooperation & $2.7165 \mathrm{~s}$ \\
$t_{c, c y c}^{c o o p}$ & Frame cyclic period with cooperation & - \\
\hline \hline \multicolumn{3}{|c|}{ Short Range Parameters } \\
\hline Character & Mean & Value \\
\hline$P_{s r, t x}$ & transmission power \\
$P_{s r, r c}$ & reception power & - \\
$P_{s r, i}$ & power consumption for idle state & $10 \mathrm{~mW}$ \\
$t_{s r, t x}$ & transmission time & $1 \mathrm{~mW}$ \\
$t_{s r, r c}$ & reception time & - \\
$t_{s r, i}$ & idle time & - \\
$t_{s r, c y c}^{c o o p}$ & cyclic period & - \\
\hline \multicolumn{2}{|}{}
\end{tabular}

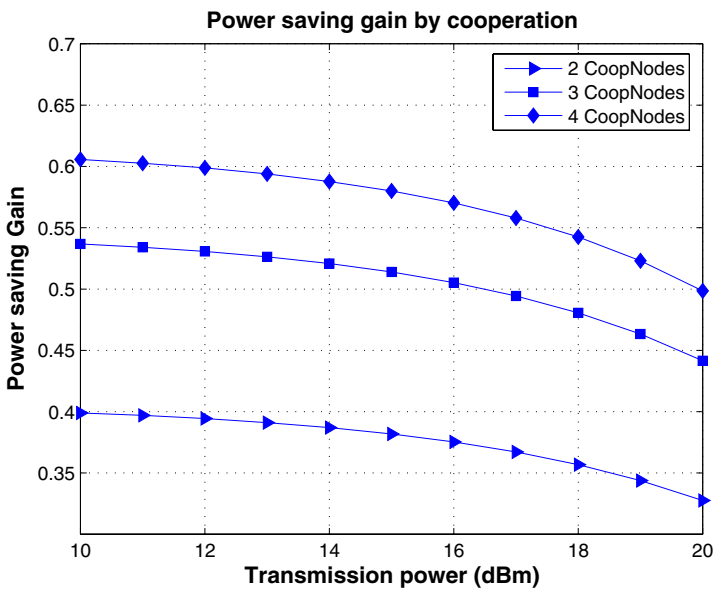

Fig. 3. Power saving gain by Cooperation

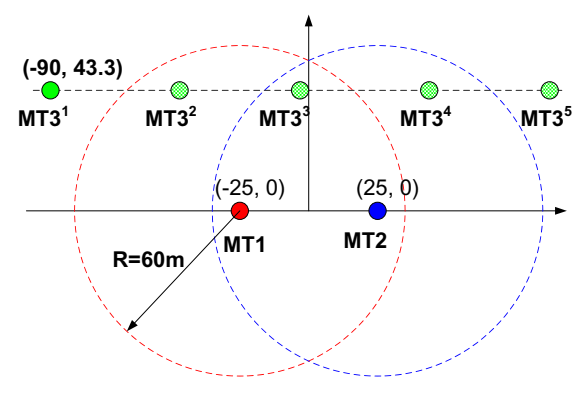

Fig. 4. Scenario of example II illustration diagram

TABLE II

PARAMETERS OF EXAMPLE II

\begin{tabular}{|l|c|l|l|l|l|}
\hline Parameter & Velocity of MT3 & $P_{s r, t x}$ & $P_{s r, r x}$ & $P_{s r, i}$ & Time \\
\hline Value & $1 \mathrm{~m} / \mathrm{s}$ & $10 \mathrm{~mW}$ & $10 \mathrm{~mW}$ & $1 \mathrm{~mW}$ & $180 \mathrm{~s}$ \\
\hline
\end{tabular}




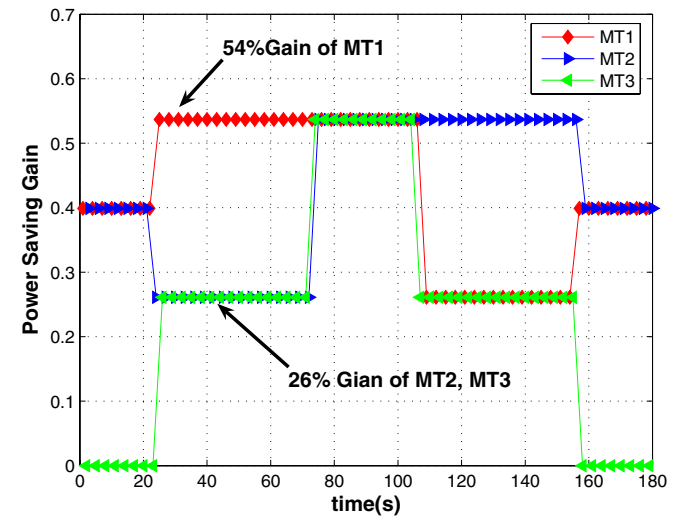

(a) equal exchanged packets

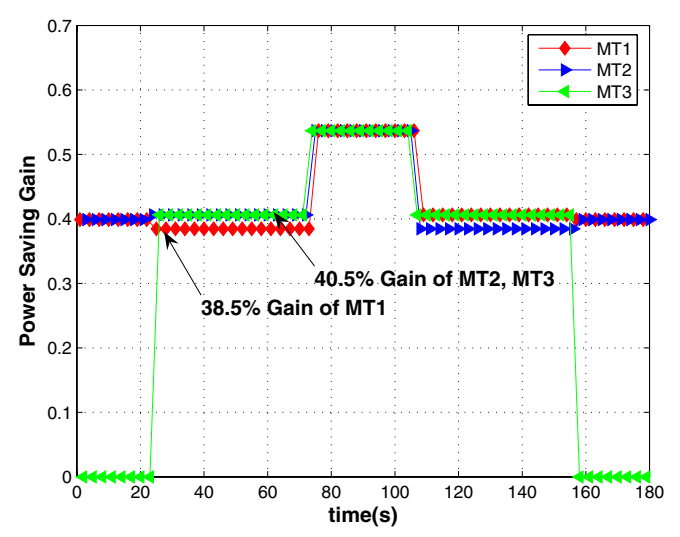

(b) Balanced Power Consumption

Fig. 5. Power Saving gain in Example II

requirement of the system. So it is based on the principle that the exchanged packets between nodes must be equal. At non-fully cooperative situation, MT1 can have about 54\% power saving gain while MT2 and MT3 only get about 26\% power saving gain (MT1 is the final winner because its optimal location). At this situation, nodes get unequally cooperative gain and the gain of MT1 is more than double of the achievable gain of MT2 and MT3. It is obvious in the Cooperation Strategy I that the gain achieved by the node depends on its relative position in the cooperative group.

Fig. 5(b) is based on the Cooperation Strategy II. This strategy tries to balance the power saving gain of the nodes, while it is not dependent on the nodes' relative location any more. Here we assume that the power consumption on the SRL is very low. Hence, if the master receives half of all DVB-H burst packets and each slave receives the remaining packets, it is very close to the optimum value to balance the power saving gain of the nodes. By this strategy at non-fully cooperative situation MT1 gets $38.5 \%$ power saving gain and MT2 and MT3 achieve $40.5 \%$ power saving gain. Note that it is obtained a very good balance of power saving gains for all nodes at a little expense of the MT1's power saving gain. Furthermore, in this case the Cooperative Strategy II saves 7.1\% more power from the standpoint of the whole cooperative system $(10 \%$ theoretically). If one master cooperates with more slaves it can save even more power by Cooperative Strategy II than Strategy I. Theoretically, it can save 20\%, 26.47\%, 30.77\% more power respectively, when one master cooperates with 3 , 4, 5 slaves $^{5}$ in one piconet.

Fig.5(a) and Fig. 5(b) also clearly show the corresponding power saving gain changing with the movement of MT3 when the different topology based cooperative algorithms are used. It is apparent that mobile terminals all achieve the maximum power saving gain when they are fully-connected. It is up to $54 \%$ in this example scenario.

\section{CONClusion}

The proposed cooperative mechanisms are used for multiinterface DVB-H terminals. Multiple air interface terminal is already a reality. Results show the strength of non-altruistic cooperation between mobile devices for IP-services over DVB$\mathrm{H}$ to save power. The numerical power consumption analysis examples show the achievable power gain by cooperative strategy using the state-of-the-art technology. The gain of power saving depends on the number of cooperative peers and the power consumption overhead on the short-range link. It is expected that data rates supported by SRL will increase significantly while power consumption in such systems will continue to decrease. Such short-range systems include UWB Bluetooth 3.0, wireless USB and others. Then the power consumption overhead on SRL will become much less and hence more power saving gain can be achieved by the proposed cooperative strategy.

\section{REFERENCES}

[1] Frank H.P. Fitzek and Marcos D.Katz, editors. Cooperation in Wireless Networks: Principle and Applications, Springer. ISBN-10 1-4020-4710X. 2006.

[2] DVB-H Implementation Guidelines, DVB-H Document A092, July 2005,

[3] Jaap C. Haartsen, "The Bluetooth Radio System", IEEE Personal Commun. Vol.7, Issue.1, pp.28-36, February 2000.

[4] Yang, X.D. and Song, Y.H. and Owens, T.J. and Cosmas, J. and Itagaki, T., "Performance analysis of time slicing in DVB-H", IEEE Mobile Future, the Symposium on Trends in Communications. SympoTIC '04. Joint IST Workshop pp.183-186, 2004.

[5] Cedric Paillard, "DVB-H could be the next big thing", http://www.commsdesign.com/showArticle.jhtml?articleID=184400015

[6] de Diego Balaguer, E. and Fitzek, F.H.P. and Olsen, O. and Gade, M., "Performance Evaluation of Power Saving Strategies for DVB$\mathrm{H}$ Services using adaptive MPE-FEC Decoding", PIMRC 2005. IEEE 16th International Symposium, Vol.4, pp.2221-2226, 2005.

[7] "Specification of Bluetooth System", Covered Core Package verison:2.0+EDR, Nov. 2004

[8] IST Project IST-2002-507014, http://dea.brunel.ac.uk/instinct/Docs/ Deliverable4.2_PublicDocs_v1-0.pdf

[9] Salonidis T.; Bhagwat P., Tassiulas L., LaMaire R.; "Distributed Topology Construction of Bluetooth Wireless Personal Area Networks", IEEE Journal on Selected Areas in Communications, Vol.23 Issue.3, pp. 633-643, 2005

[10] Changlei Liu, Yeung K.L.; "Autonomous proximity awareness of bluetooth devices", ICC 2005, Vol.2, pp. 934-938

\footnotetext{
${ }^{5}$ In such case, the maximum cooperative slaves can only reach 5 , because when more than 5 slaves are in the same piconet, two or more slaves must be in each others' cooperation range.
} 